\title{
Electric Field Control of Magnon Power Flow in Thin Ferromagnet Films
}

\begin{abstract}
V.N. KrivoruchKo* And A.S. SAVChEnKO
Donetsk Institute for Physics and Engineering, the NAS of Ukraine, 46, Nauki Av., 03028, Kyiv, Ukraine

External electric field can modify the strength of the spin-orbit interaction between spins of ions in magnetic crystals. This influence leads to a spin wave frequency shift that is linear in both the applied electric field and the wave vector of the spin wave. Here, we explore theoretically the external electric field as a means of control of the spin wave power flow in ultrathin ferromagnets. The spin wave group velocity and focusing pattern is obtained from the slowness (isofrequency) curves by evaluating their curvature at each point of the reciprocal space. We show that the combination of the magneto-dipole interaction and the electric field can result in non-reciprocal unidirectional caustic beams of dipole-exchange spin waves. Our findings open a novel avenue for spin wave manipulation and development of electrically tuneable magnonic devices.
\end{abstract}

DOI: 10.12693/APhysPolA.133.463

PACS/topics: 75.30.Ds, 42.25.Bs, 75.30.Gw

\section{Introduction}

The possibility of an electric field control of spin waves is well known [1-3]. If the crystal symmetry of a simple ferromagnet contains the spatial inversion element, the external electric field induces a frequency shift that is linear in both the applied electric field and the wave vector of the spin wave [1-3]. Microscopically, the shift originates from the spin-orbit interaction. To linear order in the electric field, this effect is equivalent to adding a Dzyaloshinskii-Moriya-like (DM-like) interaction between the spins $\boldsymbol{S}_{i}$ and $\boldsymbol{S}_{j}$ of neighboring ions and can be written in a traditional form as $\boldsymbol{d}_{i j}\left(\boldsymbol{S}_{i} \times \boldsymbol{S}_{j}\right)$, where $\boldsymbol{d}_{i j}$ is the vector perpendicular to both the electric field $\boldsymbol{E}$ and the unit vector $\boldsymbol{e}_{i j}$ along the line that connects the magnetic ions [1-3]. Initially, the frequency shift was expected to be very small $(\sim 0.01 \%)$. However, more recent theoretical [2,3] and experimental [4] studies showed an efficient electrical tuning of spin waves in thin magnetic films, such as YIG $\left(\mathrm{Y}_{3} \mathrm{Fe}_{5} \mathrm{O}_{12}\right)$.

Here, we apply these findings to examine theoretically a possibility of the electric field control of the spin wave propagation and focusing in ultrathin magnetic films. In particular, we demonstrate that the unidirectional spin wave caustic beams predicted in [5] to exist in systems with the DM interaction can also be observed in more standard magnetic systems experiencing an out-of-plane electric field. In Sec. 2, we analyze the spin-wave dispersion and the shape of the slowness (isofrequency) curves, i.e. curves of constant $\omega$ in $k$-space, with and without the electric field. In Sec. 3, the spin wave focusing effect and formation of the electrically tuneable caustics are discussed. Numerical examples are presented for an ultrathin ferromagnetic film with perpendicular magnetic anisotropy and different magnitudes of the electric field.

*corresponding author; e-mail: krivoruc@gmail.com

\section{Dispersion of spin waves in electric field}

Let us consider an ultrathin magnetic film located in the $x-y$ plane. An in-plane magnetic field $\boldsymbol{H}_{0}$ applied parallel to the $\boldsymbol{y}$ axis is strong enough to saturate the equilibrium magnetization of the film, $\boldsymbol{M} \| \boldsymbol{H}_{0}$. We focus our attention to films of a few nanometer thickness, so that only a single spin-wave branch with amplitude uniform across the film thickness needs to be considered. Taking into account the magneto-dipole and exchange interactions, the Zeeman energy associated with the applied magnetic field and the effective uniaxial (shape) anisotropy, the frequency of the main mode is given by (see e.g. $[6,7]$ ):

$$
\omega_{0}(k)=\gamma \mu_{0}\left[H_{x}(k) H_{y}(k)\right]^{1 / 2} .
$$

Here, $\gamma$ is the gyromagnetic ratio, $\mu_{0}$ is the vacuum permeability, and

$$
\begin{aligned}
& H_{x}\left(k_{\|}\right)=H_{0}+D k_{\|}^{2}+M_{S} d k_{x}^{2} / 2 k_{\|}, \\
& H_{y}\left(k_{\|}\right)=H_{0}+D k_{\|}^{2}-H_{K}-M_{S}\left(k_{\|} d / 2\right),
\end{aligned}
$$

where $M_{S}$ is the saturation magnetization, $D=$ $2 A / \mu_{0} M_{S}, A$ is the exchange constant, $d$ is the film thickness, the wave vector $\boldsymbol{k}$ is in the $x-y$ plane, $k_{\|}=$ $\left(k_{x}^{2}+k_{y}^{2}\right)^{1 / 2}$, and $H_{K}=\left(2 K_{u}-\mu_{0} M_{S}^{2}\right) / \mu_{0} M_{S}$ is the effective anisotropy field, where $K_{u}$ is the interface-induced perpendicular magnetic anisotropy. Here we take into account that (i) in the thin-film limit $k_{\|} d \ll 1$ the dominant contribution to the dipole field is linear in $k_{\|}$[7], and (ii) the angle $\varphi_{k}$ between the magnon wave vector $\boldsymbol{k}$ and the $\boldsymbol{y}$ axis is $\sin \varphi_{k}=k_{x} / k_{\|}$.

As shown in [1-4], the electric field can modify the spin-wave dispersion. Just as a magnetic field shifts the dispersion vertically by increasing or decreasing the frequency at fixed $\boldsymbol{k}$, the electric field shifts the dispersion horizontally by increasing or decreasing the wave vector at fixed frequency [1-4]. For a geometry in which the wave vector $(\boldsymbol{k} \| \boldsymbol{x})$, the magnetization $(\boldsymbol{M} \| \boldsymbol{y})$ and the electric field $(\boldsymbol{E} \| \boldsymbol{z})$ are mutually orthogonal, the largest 
relative wave vector shift is observed for the lowest lying modes. Therefore, we apply the electric field normal to the $x-y$ plane and consider spin-wave propagation in this plane. Then, in accordance with [1-3], the spin wave frequency (1) acquires a shift, so that the modified dispersion relation becomes

$$
\omega(k, E)=\omega_{0}(k)-\omega_{E}(k),
$$

where $\omega_{E}(k)=\omega_{M} \lambda_{S O} E k_{x}, \lambda_{S O}=\frac{4 A}{\mu_{0} M_{S}^{2}} \frac{|e|}{a E_{S O}}, \omega_{M}=$ $\gamma \mu_{0} M_{S}$, and $E_{S O}$ is an energy scale that is inversely proportional to the spin-orbit interaction strength (for YIG $\left.E_{S O} \sim 1 \mathrm{eV}[3,4]\right)$. Thus, the external electric field adds to the spin-wave frequency a contribution that depends linearly on the wave vector and makes the spin-wave propagation nonreciprocal $\omega(\boldsymbol{k}, \boldsymbol{E}) \neq \omega(-\boldsymbol{k}, \boldsymbol{E})$. Without the electric field, the curves of constant spin-wave frequency $\omega$ in $\boldsymbol{k}$-space ("isofrequency contours") form an ellipse centered at the origin. However, the electric field through the last term in Eq. (2) pushes the isofrequency contours in the direction parallel to $(\boldsymbol{E} \times \boldsymbol{M})$.

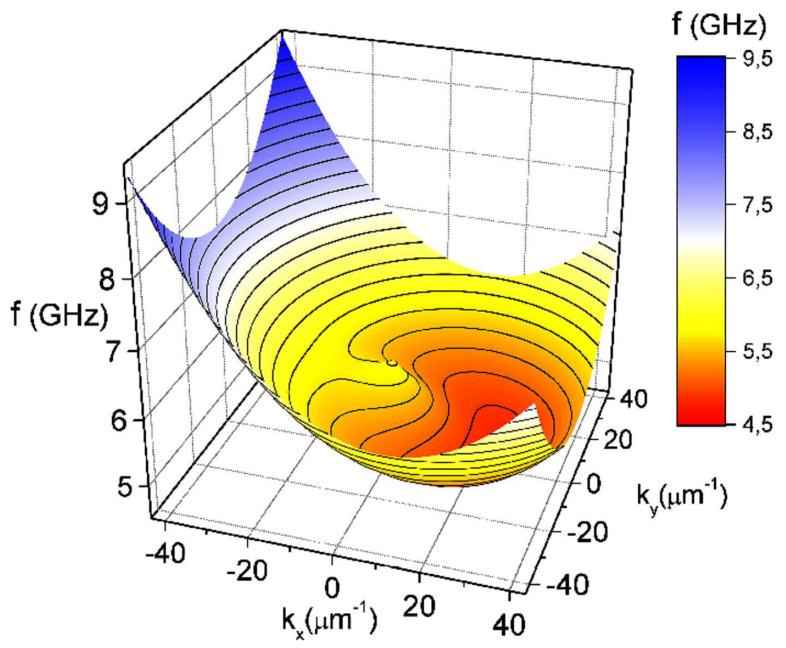

Fig. 1. 3D spin waves frequency contours $\omega / 2 \pi=f$, Eq. (2), at $E=3 \times 10^{7} \mathrm{~V} / \mathrm{m}$; each successive contour represents a frequency difference $\Delta f=0.2 \mathrm{GHz}$. Here and in Fig. 2, the numerical results are obtained for ultrathin ferromagnetic film with perpendicular magnetic anisotropy and material parameters close to Permalloy. Namely: $d=2 \mathrm{~nm}, M_{S}=1 \mathrm{MA} / \mathrm{m}, K_{U}=$ $1 \mathrm{MJ} / \mathrm{m}^{3}, A=15 \mathrm{pJ} / \mathrm{m}, E_{S O}=1 \mathrm{eV}, \mu_{0} H=0.8 \mathrm{~T}$, $\gamma=1.7608597 \times 10^{11} \mathrm{rad} /(\mathrm{s} \mathrm{T}) \mu_{0}=4 \pi \times 10^{-7} \mathrm{H} / \mathrm{m}$.

Figure 1 illustrates the formation of isofrequency contours for $\omega(\boldsymbol{k}, \boldsymbol{E})$ given by Eq. (2). In the case shown, the electric field of $E=3 \times 10^{7} \mathrm{~V} / \mathrm{m}$ shifts the energy minimum to $k_{x}=25.37 \mu \mathrm{m}^{-1}$ and the lowest frequency is detected at $\omega / 2 \pi=f=4.5 \mathrm{GHz}$. For spin waves in thin films with in-plane magnetization, the dispersion relation is inherently anisotropic. Application of the external electric field results in additional nonreciprocity of these waves. In the next section, we discuss how the nonreciprocity in the spin wave propagation caused by an external electric field leads to spin wave power focusing and caustics beams.

\section{Propagation of spin waves under an electric field}

One of the requirements for wave focusing is that there is some anisotropy in the system so that the group velocity and the phase velocity point in different directions. In anisotropic medium, the group velocity $\boldsymbol{v}_{g}$ and wave vector $\boldsymbol{k}$ are noncollinear, in general. In this case, a situation is possible when the energy transferred to anisotropic medium from a point excitation source transfers away not in circular waves but in focused directions, forming so-called caustic wave beams $[8,9]$. The focusing direction can be found from the isofrequency curves (curves of constant $\omega$ in $\boldsymbol{k}$-space) by calculating the normal to the curve (the group velocity, $v_{g}=\partial \omega(k, E) / \partial k$ ) at each point. These normals indicate the directions of the energy flow. The magnitude of the energy sent in that direction is proportional to the square root of the curvature of the surface at a given point. When the curvature is zero, one finds the so-called caustics beams, when for waves having different wave vectors $\boldsymbol{k}$ the group velocity is the same and, formally, in those directions the power flow diverges [8-11].

From Eq. (2) it is evident that the spin-wave dispersion is anisotropic and depends on direction of the in-plane wave vector with respect to the direction $(\boldsymbol{E} \times \boldsymbol{M})$. For the group velocity, we have

$$
\boldsymbol{v}_{g}=\partial \omega_{0}(\boldsymbol{k}) / \partial k-\omega_{M} \lambda_{S O} E \boldsymbol{e}_{x}
$$

and thus, a difference in the $\boldsymbol{v}_{g}$ between the left and right with respect to $k_{x}=0$ slowness surfaces is simply proportional to the applied field.

In Fig. 2, we illustrate how the isofrequency curves change as the electric field is applied and nonreciprocity in spin wave propagation becomes pronounced. We present the frequency contours of the dispersion relation Eq. (3) for zero electric field (the top panel) and at $E=2 \times 10^{7} \mathrm{~V} / \mathrm{m}$ (the bottom panel) with the energy step $\Delta f=0.2 \mathrm{GHz}$. The group velocity patterns, the normals to the isofrequency curves, are shown in Fig. 2 (the red lines with arrows), respectively. The existence of three slowness surfaces in $E=0$ results in interference for all propagation directions at low frequencies. At higher frequencies, the exchange term $D k_{\|}^{2}$ becomes dominant and the isofrequency curves recover a more circular shape and is positive everywhere. Under the influence of external electric field, the spin wave dispersion minimum is shifted from its position at $k_{x} \approx 18.5 \mu \mathrm{m}^{-1}$ in $\boldsymbol{k}$ space at $E=0$ up to $k_{x} \approx 23.3 \mu \mathrm{m}^{-1}$ at $E=2 \times 10^{7} \mathrm{~V} / \mathrm{m}$. Application of a weak electric field $E \sim 10^{7} \mathrm{~V} / \mathrm{m}$ results in a coupling of two slowness surfaces, $5.3 \mathrm{GHz}$ contours in $E=0$, into one. Also, in the isofrequency curves a 'dent' along the $-k_{x}$ axis appears for low frequencies. The presence of the dent indicates that the curvature of the isofrequency curves changes sign, which means that caustics are created.

Some evidence of interference can already by seen in Fig. 2, $E=2 \times 10^{7} \mathrm{~V} / \mathrm{m}$, for $5.3 \mathrm{GHz}$ in the region bounded by the two focused beams. As it can be seen 

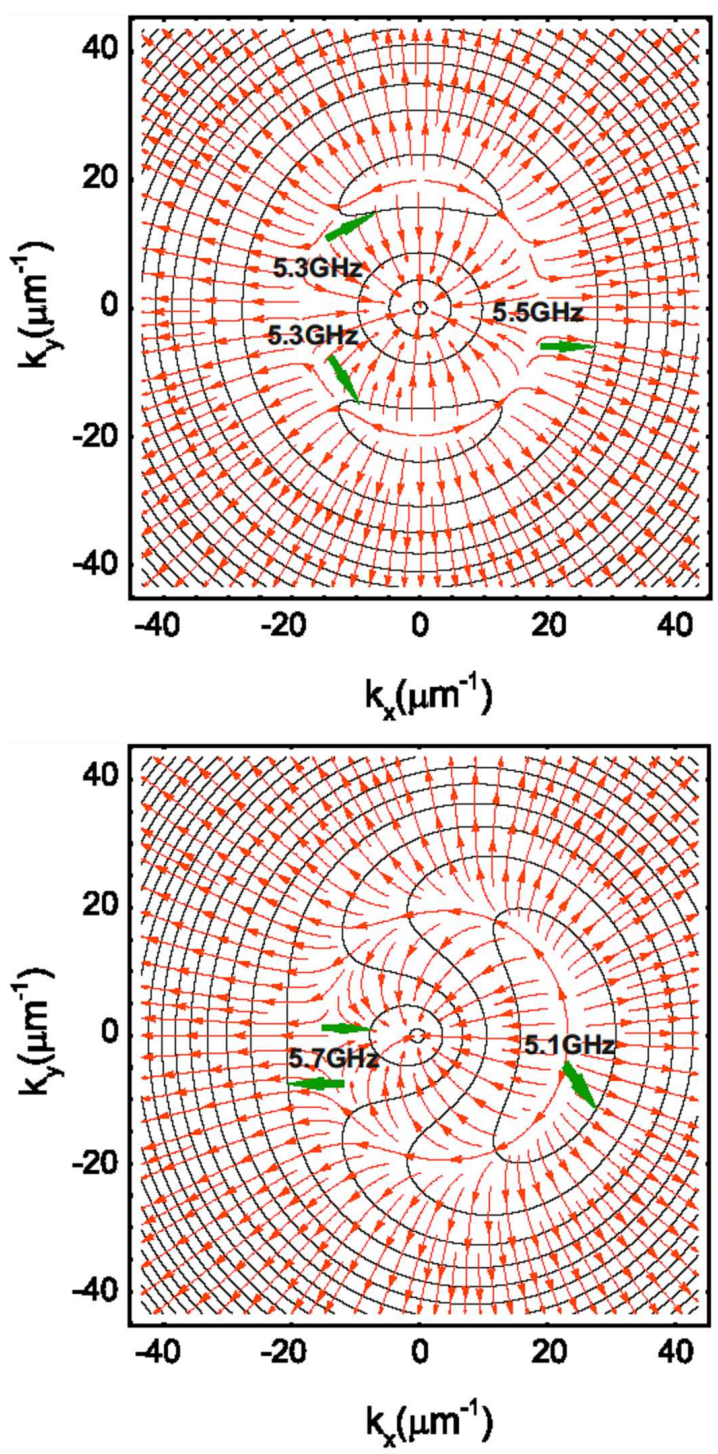

Fig. 2. Isofrequency contours (the black curves) determined from Eq. (2) and the group velocity (the red lines with arrows) determined from Eq. (3) at different frequencies. The top panel: $E=0$, the bottom panel: $E=2 \times 10^{7} \mathrm{~V} / \mathrm{m}$. The group velocity, the normal to the isofrequency curve, is indicated along each isofrequency curve. At $\boldsymbol{k}=(0,0) f=5.9 \mathrm{GHz}$, each successive contour represents a frequency difference $\Delta f=0.2 \mathrm{GHz}$. Some reference frequencies are indicated by green arrows.

from the figure, the frequency contour at $5.5 \mathrm{GHz}$ demonstrates a C-shaped isofrequency curve. If we examine how the group velocity vector, $\boldsymbol{v}_{g}$, evolves around this surface, we notice that certain orientations of $\boldsymbol{v}_{g}$ appear at multiple points along this surface, which indicates that propagation along these directions involves partial waves with different $\boldsymbol{k}$. The focusing patterns at finite $\boldsymbol{E}$, shown in Fig. 2 determine the group velocity direction in each point and, in general, are not collinear with that of the wave vector. This is a direct consequence of the anisotropy of the dispersion relation.

\section{Conclusion}

Possibility to control the spin wave group velocity is an important factor for magnonic device design, where the transport of angular momentum and energy both play a key role. In this report, we study the spin wave power flow in a ferromagnetic film under an electric field. Without an external electric field, in the ultrathin film limit, the power flow is essentially isotropic, radiating energy approximately equally in all directions. When an electric field applied, we find a few remarkable results. It is shown that in the electric field one can create caustics, highly focused beams of energy flow, at particular frequencies. The focusing patterns are nonreciprocal, with the caustic beams appearing only on one side of the film. This has important implications for magnonic devices, where the transfer of angular momentum and energy play a key role. Thus, it is demonstrated that an external electric field is an energy-efficient way to control the spin current propagating in an insulating magnet films. These findings open a novel important avenue for spin wave manipulation and development of electrically tuneable magnonic devices [12].

\section{Acknowledgments}

This work was supported by the MagIC project: 644348-H2020-MSCA-RISE-2014 and the Ukrainian State Fund for Fundamental Research (project: F71/592017). The authors acknowledge fruitful discussions with V. V. Kruglyak from the University of Exeter.

\section{References}

[1] D. L. Mills, I.E. Dzyaloshinskii, Phys. Rev. B 78 184422 (2008).

[2] T. Liu, G. Vignale, Phys. Rev. Lett. 106, 247203 (2011).

[3] T. Liu, G. Vignale, J. Appl. Phys. 111, 083907 (2012).

[4] X. Zhang, T. Liu, M.E. Flatté, H.X. Tang, Phys. Rev. Lett. 113, 037202 (2014).

[5] J.-V. Kim, R.L. Stamps, R.E. Camley, Phys. Rev. Lett. 117,197204 (2016).

[6] B.A. Kalinikos, A.N. Slavin, J. Phys. C: Solid State Phys 19, 7013 (1986).

[7] R. Arias, D.L. Mills, Phys. Rev. B 60, 7395 (1999).

[8] A.V. Vashkovsky, E.H. Lock, Phys. Usp. 49, 389 (2006).

[9] E.H. Lock, Phys. Usp. 51, 375 (2008).

[10] V. Veerakumar, R. E. Camley, Phys. Rev. B 74, 214401 (2006).

[11] T. Schneider, A.A. Serga, A.V. Chumak, C.W. Sandweg, S. Trudel, S. Wolff, M.P. Kostylev, V.S. Tiberkevich, A.N. Slavin, B. Hillebrands, Phys. Rev. Lett. 104, 197203 (2010).

[12] V.V. Kruglyak, S.O. Demokritov, D. Grundler, J. Phys. D: Appl. Phys. 43, 264001 (2010). 